\title{
Relationship between behavioral indices of pain during labor pain with pain intensity and duration of delivery
}

\author{
Bibi Marziyeh Hashemi Asl ${ }^{1}$, Atiyeh Vatanchi ${ }^{2}$, Nahid Golmakani ${ }^{3}$, Alireza Najafi ${ }^{4}$
}

\author{
${ }^{1}$ Samen Health Center, Mashhad University of Medical Sciences, Mashhad, Iran \\ ${ }^{2}$ Assistant Professor of Obstetrics and Gynecology, Department of Obstetrics and Gynecology, Faculty of Medicine, \\ Mashhad University of Medical Sciences (MUMS), Mashhad, Iran \\ ${ }^{3}$ Assistant Professor, Department of Nursing and Midwifery, Mashhad University of Medical Sciences, Mashhad, \\ Iran \\ ${ }^{4}$ B.Sc. of Physiotherapy, Ghaem Hospital, Mashhad University of Medical Sciences, Mashhad, Iran
}

Type of article: Original

\begin{abstract}
Background: Certain behaviors can be adopted by women to cope with labor pain according to their individual characteristics, which are currently called behavioral indicators during labor pain, and include facial expressions, verbal expressions, tone of voice, body movements, degree of relaxation, and respiratory system functioning during delivery. Moreover, severity of pain and duration of labor can vary due to several factors including individual characteristics.

Objective: The purpose of the present study was to determine the relationship between behavioral indicators during labor pain, severity of pain, and delivery duration.

Methods: In this cross-sectional study, 120 low risk pregnant women who referred to Omolbanin (AS) Hospital in the city of Mashhad (Iran) for delivery in 2014, were selected via convenience sampling method, which was then followed by completion of demographic information forms. From cervical dilatation of 3-5 centimeters until delivery, the Labor Pain Coping Behavior Observation Form (comprised of 6 sub-groups of facial expressions, verbal expressions, tone of voice, body movements, degree of relaxation, and respiratory function and severity and duration of pain) was completed during uterine contractions and every half an hour. Using the Inventory of Labor Information; vital signs, frequency of contractions, and duration of the first and second stages of labor were measured. Furthermore, the content validity of the questionnaire was determined and its reliability was confirmed by Cronbach's alpha method. Then, the data were analyzed using the SPSS version 16, through Pearson Product-Moment Correlation and Spearman's Rank-Order Correlation, Kruskal-Wallis test, and ANOVA.

Results: According to the results, $16.2 \%$ of the individuals had undesirable behavioral indicators during labor pain, $50 \%$ of them were endowed with acceptable behaviors, and $33.8 \%$ of these women had desirable behaviors. The findings also revealed that the duration of the active phase of the first stage of labor $(\mathrm{p}<0.001$ and $\mathrm{r}=-0.453)$, the duration of the second stage of labor $(\mathrm{p}<0.012$ and $\mathrm{r}=-0.146)$, and the severity of pain $(\mathrm{p}<0.001$ and $\mathrm{r}=-0.450)$ were significantly and inversely correlated with behavioral indicators during labor pain; i.e. an increase in the mean score of behavioral indicators during labor pain could lead to a decline in the duration of stages of labor and severity of pain.

Conclusion: It was concluded that behaviors demonstrated by women in labor had effects on their pains in the course of delivery, and there was also a relationship between the duration of stages of labor and its severity of pain. Therefore, it was recommended to turn attention to behaviors by women in labor in order to achieve a desirable clinical management.
\end{abstract}

Keywords: Coping behavior, Labor pain, Pain intensity

\section{Corresponding author:}

Assistant Professor Dr. Atiyeh Vatanchi, Department of Obstetrics and Gynecology, Faculty of Medicine, Mashhad University of Medical Sciences (MUMS), Mashhad, Iran.

Tel.: +98.9153106837, Fax:+98.5138430569, Email: VatanchiA@mums.ac.ir

Received: July 27, 2016, Accepted: August 12, 2017, Published: January 2018

iThenticate screening: August 14, 2017, English editing: December 21, 2017, Quality control: January 12, 2018

(C) 2018 The Authors. This is an open access article under the terms of the Creative Commons Attribution-NonCommercialNoDerivs License, which permits use and distribution in any medium, provided the original work is properly cited, the use is non-commercial and no modifications or adaptations are made. 


\section{Introduction}

Labor pain results from a series of physiological factors such as uterine contractions and cervical dilatation along with mental and emotional factors including fear and anxiety. In addition, factors such as maternal age, parity, physical strength of women in labor, and maternal status can have an impact on its severity and duration (1). The amount of perceived labor pain can also widely vary in different women and it can be similarly influenced by numerous factors. In this regard, research studies have shown that further pain severity is correlated with factors such as lower age, less education, unrealistic expectations regarding pain, as well as low socio-economic status (2). Among the other factors affecting labor pain are levels of excitement and mood in individuals, such as fear and anxiety, degree of personal control over pain, prior experiences, surrounding environment quality, fetal size and position, myometrium contractility power, position of women in labor, as well as progression of cervical dilatation (3). Severe labor pains can cause emotional turmoil in mothers, disrupt her mental health, and consequently have a negative impact on relationships with her infant and partner. Moreover, a growing fear of labor pains can lead to an increase in muscle stretch and tension and consequently direct blood oxygen to the brain and muscles. Stress, fatigue, and sensitivity to pain can also have an ascending trend in mothers, and thus reduce their ability to adapt to such pains (4). Accordingly; responses to pain in women are increased, impairments are observed in the efficiency of uterine contractions, labor is prolonged, and there is a need to have obstetric interventions which can lead to some complications. As duration of labor pains is prolonged; the possibility of aspiration, infection, and physical and neurological damage to newborns also multiplies and mothers will be at risk of increased postpartum bleeding and infection as well as mental confusion due to anxiety, insomnia, and fatigue (5). It should be noted that behaviors demonstrated by individuals in the face of pain are unique (6) and they can be influenced by ethnic, psychological, cultural, social, and environmental variables associated with pain behaviors (7). In this respect; factors such as emotions as well as environmental, social, and cultural contexts together with biological aspects of pain, can play a major role in the way they react to pain. Accordingly, personal characteristics can have a strong mediating effect on psychological and physiological experiences with pain, and thus intellectual, emotional, and behavioral efforts made by individuals when encountered with psychological pressures in order to overcome, endure, or minimize side effects that can be observed through behavioral indicators of pain (8). These behaviors might be seen in the form of dominant verbal and non-verbal messages in a person suffering (9). Non-verbal messages such as vocal behaviors, changes in facial expressions, body movements, crying, fists, and body stretch not only complement verbal messages but also represent patients' real thoughts and feelings better than verbal messages. Sometimes, even verbal messages are likely to be false, while non-verbal behaviors imply real senses $(10,11)$. Likewise, the incidence of undesirable behaviors in stressful and painful situations like screaming and crying can result in increased oxygen consumption as well as physical and mental fatigue, and the side effects of non-ideal behaviors can lead to severe pains in a vicious cycle (6). With the start of labor pains, most women have a sense of loneliness and insolvency and in fact, they make their own body contracted following the most primitive contractions related to labor. During this faulty period, tension and pain grow and consequently, discomfort in women in labor goes up (12). There is little evidence about behavioral indicators in the face of labor in Iran. For example, Khorsandi et al., in their study, found that evaluating a person's ability to cope with stressful situations was a key factor in the incidence of labor behaviors (13). According to the results of the study by Rastegari et al., those endowed with higher levels of self-efficacy had experienced less fear and more satisfaction during labor pains and their higher self-efficacy had led to better behaviors, more self-confidence, and a smaller amount of pain perception (14). In the study by Abushaikha, a significant relationship was reported between fear of delivery and response to obvious pains such as screaming and crying (15). Also, in the study of Tampawiboon. (2005), women who suffered from higher levels of anxiety and more fear of delivery, demonstrated greater states of crying, screaming, and losing control (6). Although there are a lot of of research studies about the factors associated with labor pain and its non-behavioral indicators such as severity of pain and delivery duration, there is little evidence regarding behavioral indicators during labor pain and also the relationship between individuals' unique reactions and behaviors towards pain, and other features such as duration and severity of pain have been less considered. In this domain, the existing behavioral patterns in the Iranian community indicate that women in labor assume that they have to scream and demonstrate undesirable behaviors and also have little control over their pains with the onset of labor pains; therefore, they prefer Cesarean section to normal vaginal delivery. The fact is that the high rate of Cesarean section (50\%) signifies lack of comprehensive knowledge regarding factors involved in severity and duration of labor pain (16). In recent years, short-term breathing and relaxation techniques are being taught to pregnant women within Iranian universities, through holding childbirth preparation classes to help them cope with delivery, and also there is evidence on the effectiveness of such an education in reducing labor pain (14). Therefore, identifying most behavioral manifestations and indicators can influence the reduction of severity and duration of labor pains by demonstrating the type of effective behaviors moderating pain. Thus, adopting low-risk and safe methods such as making changes in coping 
behaviors, can prevent the consequences of severe labor pains and prolonged delivery. Accordingly, the purpose of this study was to determine the relationship between behavioral indicators during labor pain, severity of pain, and delivery duration.

\section{Material and Methods}

\subsection{Design and participants}

In this cross-sectional study, the sample consisted of 120 pregnant women who had referred to Omolbanin (AS) Hospital in the city of Mashhad for delivery in 2014. Based on the pilot study and considering similar research studies, the sample size was calculated by 120 individuals with a $99 \%$ degree of confidence test $(\alpha=0.01)$ and test power of $90 \%(\beta=0.1)$.

\subsection{Selection criteria}

\subsubsection{Inclusion criteria:}

The inclusion criteria were age range between 18 and 35 years, gestational age based on last menstrual period or first trimester solography, 38-40 weeks of pregnancy, writing and reading literacy, no formal education in the domain of pain relieving methods, no history of medical and mental illnesses, low-risk pregnancy, no indication of cesarean section; no hearing, speech, or accent disorders to communicate with the researcher, and no use of sedatives or herbal medicine during the last 24 hours of the onset of spontaneous labor.

\subsubsection{Exclusion criteria:}

The exclusion criteria were obstetric problems, more-than-usual supportive care for the woman in labor by midwife or another person in the delivery room, use of narcotics, Entonox, or anesthetics in the course of labor, or the signs of fetal distress and induction or augmentation of contractions.

\subsection{Instrument}

Data collection instruments included Demographic Information Form along with Obstetrical History Scale, Lifestyle Inventory, and the Labor Pain Coping Behavior Observation Form as well as questionnaires on delivery duration and severity of labor pain. The Labor Pain Coping Behavior Observation Form was comprised of 6 sub-groups and 21 items about facial expressions, verbal expressions, tone of voice, body movements, relaxation, and respiratory system functioning. The score range obtained from the questionnaires was from 7-21 and it was divided into three levels including undesirable (7-11), acceptable (12-16), and desirable (17-21) (6). The questionnaire had also been employed as a valid instrument in different research articles and theses in Thailand (17 and 18). To determine the validity of this questionnaire which was in Thai, it was firstly translated into English and then into Persian after obtaining permission from the author, and its content validity was finally confirmed. In order to check the reliability of this questionnaire, Cronbach's alpha method was also used and the reliability values of $0.94,0.92,0.89,0.92$, 0.93 and 0.90 were obtained for each sub-group including facial expressions, verbal expressions, tone of voice, body movements, degree of relaxation, and respiratory system functioning; respectively.

\subsection{Data collection}

Convenience sampling method was used in this study, and then Demographic Information Form, Obstetrical History Scale, and Lifestyle Inventory were completed. The Labor Pain Coping Behavior Observation Form along with questionnaires on severity and duration of labor pain were also completed in the active phase (dilatation of 3-5 centimeters) to delivery every 30 minutes during uterine contractions. The controls during the active phase related to the frequency of uterine contractions, heart rate monitoring, and vaginal examinations were similarly performed and recorded every hour. Vital signs at the time of admission and every 2 hours after it were also monitored and recorded. Additionally, information related to the second stage of labor including the duration of the second phase of labor was recorded by the researcher.

\subsection{Statistical analysis}

After collecting the data, they were analyzed using the SPSS version 16 (SPSS Inc., Chicago, Illinoi, USA) and statistical tests of Kruskal-Wallis, ANOVA, Pearson Product-Moment Correlation, and Spearman's Rank-Order Correlation.

\subsection{Ethics of research}

Ethical considerations in all stages of the study included obtaining written permission from the Ethics Committee of the Vice-Chancellor's Office for Research at Mashhad University of Medical Sciences (Code Number: 89991) and an official letter of introduction from the Mashhad School of Nursing and Midwifery to conduct the research, to 
provide references, and to explain the purpose of the study to authorities in Omolbanin Hospital and to obtain their approval. Informed consent forms were also signed by the participants, who had been informed that they had rights to withdraw from the study. Likewise; the researcher tried to answer questions raised by the participants and also assure them of confidentiality of data and its presentation in the form of figures and statistics in the group. Other ethical issues were similarly observed during the development of the present study.

\section{Results}

The mean age of the study participants was 25.1 years with a standard deviation of 4.9 years and these individuals were between the ages of 18 and 35. In this study, the general characteristics of all the participants were also studied (Table 1). Also, the behavioral indicators during labor pain were divided into desirable, acceptable, and undesirable levels. Based on the results of the study, $73.7 \%$ of the individuals were endowed with acceptable and desirable behavioral indicators during labor pain. According to the results of this study, the mean score for behavioral indicators during labor pain was equal to $15.1 \pm 2.7$ with the minimum score of 9 and the maximum score of 20.6 which was calculated for each person. The behavioral indicators during labor pain were also recorded at least 4 times and with the maximum of 12 times from dilatation of 3-5 centimeters to delivery (every half an hour). The difference between 4 and 12 times of recordings was due to the variation during the active phase of the first stage of labor in participants and it included 6 sub-groups of facial expressions, verbal expressions, tone of voice, body movements, degree of relaxation, and respiratory system functioning (Table 2).

Table 1. The frequency of some individual variables, midwifery history and lifestyle of participants

\begin{tabular}{|c|c|c|c|}
\hline \multicolumn{2}{|l|}{ Variable } & $\mathrm{n}$ & $\%$ \\
\hline \multirow[t]{3}{*}{ Gravidity } & First Pregnancy & 56 & 46.7 \\
\hline & Second Pregnancy & 39 & 32.5 \\
\hline & Third pregnancy and more & 25 & 20.8 \\
\hline \multirow[t]{2}{*}{ Parity } & Primiparous & 62 & 51.7 \\
\hline & Multiparous & 58 & 48.3 \\
\hline \multirow[t]{3}{*}{ Employment } & Housewife & 111 & 92.5 \\
\hline & Student & 3 & 2.5 \\
\hline & Employee & 6 & 5 \\
\hline \multirow[t]{4}{*}{ Exercise (per day) } & Not at all & 91 & 75.8 \\
\hline & Less than two hours & 19 & 15.8 \\
\hline & 2-4 hours & 7 & 5.8 \\
\hline & More than 4 hours & 3 & 2.5 \\
\hline \multirow[t]{3}{*}{ leisure time (per week) } & Less than 4 hours & 89 & 74.2 \\
\hline & 4-8 hours & 28 & 23.3 \\
\hline & More than 8 hours & 3 & 2.5 \\
\hline \multirow[t]{3}{*}{ Religious rituals (hours/day) } & $<0.5$ & 18 & 15 \\
\hline & $0.5-1.5$ & 76 & 63.3 \\
\hline & $>1.5$ & 26 & 21.7 \\
\hline \multirow[t]{4}{*}{ prenatal care } & Regular & 103 & 85.9 \\
\hline & Irregular & 16 & 13.3 \\
\hline & Acceptable & 60 & 50 \\
\hline & Favorable & 40 & 33.3 \\
\hline
\end{tabular}

Table 2. The mean of Pain Behavioral Indicators Score divided to subgroups in primiparous and multiparous

\begin{tabular}{|l|l|l|l|}
\hline \multirow{2}{*}{ Pain Behavioral Indicators Score } & Mean (SD) & \multicolumn{2}{|l|}{} \\
\cline { 2 - 4 } & Primipara $(\mathrm{n}=59)$ & Multipara $(\mathrm{n}=61)$ & Total $(\mathrm{n}=120)$ \\
\hline Facial expression & $4.1(0.8)$ & $4.1(0.9)$ & $4.1(0.9)$ \\
\hline Verbalization & $2.1(0.5)$ & $2(0.5)$ & $2.1(0.5)$ \\
\hline Tone of voice & $2.2(0.5)$ & $2.1(0.5)$ & $2.2(0.5)$ \\
\hline Body movement & $2.2(0.5)$ & $2.1(0.4)$ & $2.2(0.5)$ \\
\hline Degree of Relaxation & $2.1(0.5)$ & $2.1(0.5)$ & $2.1(0.5)$ \\
\hline Control of breathing & $2.5(0.4)$ & $2.4(0.5)$ & $2.4(0.4)$ \\
\hline Mean of Pain Behavioral Indicators Score & $15.3(2.6)$ & $14.9(2.9)$ & $15.1(2.7)$ \\
\hline
\end{tabular}


Although the mean scores of the behavioral indicators during labor pain were different in nulliparous and multiparous women, no significant difference was observed in this respect $(p>0.05)$. The severity of labor pain was also measured every half an hour from dilatation of 3-5 centimeters to delivery using Visual Analogue Scale and through asking direct questions from the patients. The mean of labor pain scores was 81.4. In this regard, the highest score was 100 and the lowest one was 58.7. With regard to the correlation between behavioral indicators during labor pain and pain severity using Spearman's rank-order correlation $(\mathrm{p}<0.001$ and $\mathrm{r}=-0.450)$, linear regression analysis was used to predict the changes in labor pain severity scores for the mean score of variations in behavioral indicators during labor pain (Figure 1).

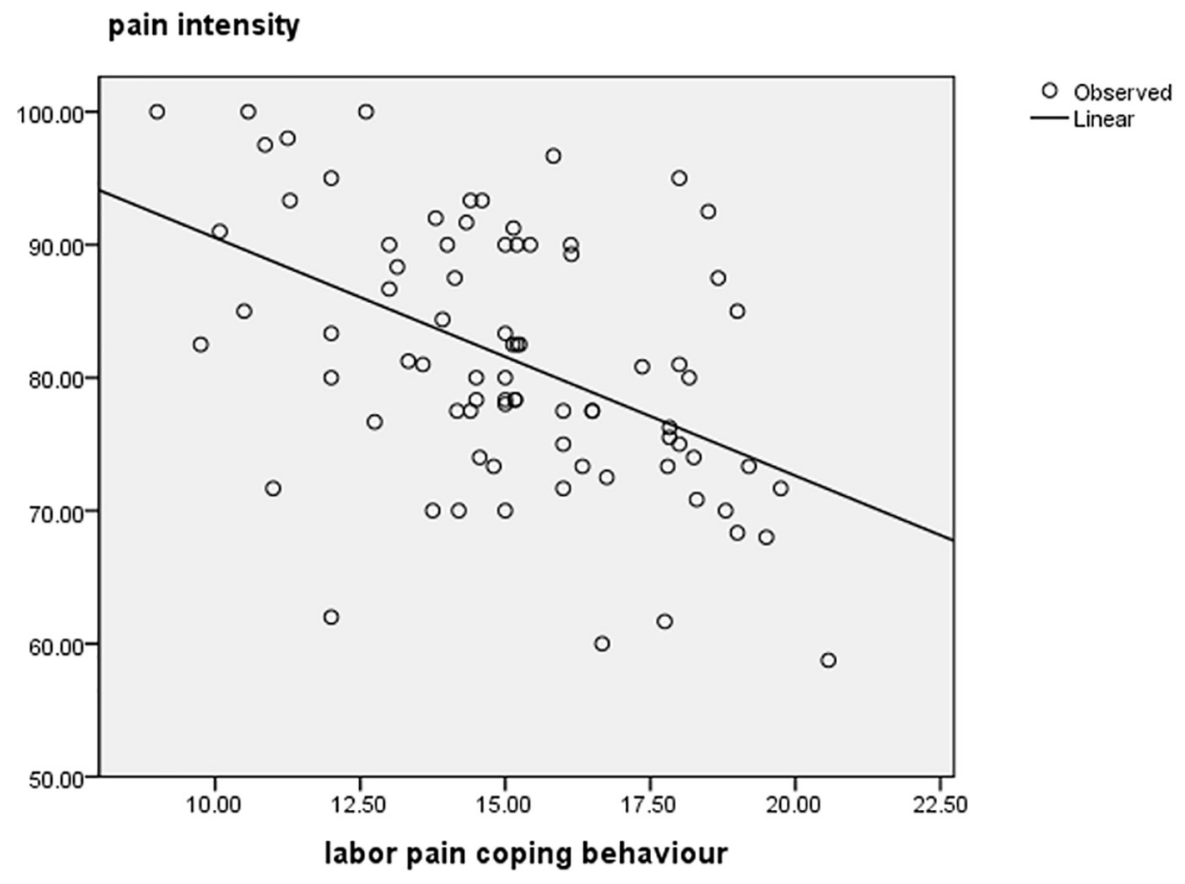

Figure 1. Relationship between mean scores of behavioral indicators during labor pain and severity of labor pain

Table 3. The mean of Pain Behavioral Indicators Scores divided to the active phase length of the first stage in primiparous and multiparous

\begin{tabular}{|l|l|l|l|l|}
\hline The active phase length of the first stage & Delivery Rating & \multicolumn{3}{|l|}{ Pain Behavioral Indicators Score } \\
\cline { 3 - 5 } & & $\mathrm{n}$ & Mean & SD \\
\hline \multirow{3}{*}{ Less than 3 hours } & Primipara & 1 & 18.9 & 0.8 \\
\cline { 2 - 5 } & Multipara & 6 & 17.1 & 4 \\
\cline { 2 - 5 } & Total & 7 & 17.7 & 3.3 \\
\hline \multirow{3}{*}{ Between 3 and 4 hours } & Primipara & 3 & 15.8 & 2.1 \\
\cline { 2 - 5 } & Multipara & 14 & 15.7 & 1.6 \\
\cline { 2 - 5 } & Total & 17 & 15.8 & 2 \\
\hline \multirow{3}{*}{ Between 4 and 5 hours } & Primipara & 24 & 14.9 & 2.6 \\
\cline { 2 - 5 } & Multipara & 34 & 15 & 2.3 \\
\cline { 2 - 5 } & Total & 58 & 15 & 2.4 \\
\hline \multirow{3}{*}{ Between 5 and 6 hours } & Primipara & 31 & 12.4 & 2.6 \\
\cline { 2 - 5 } & Multipara & 5 & 14.2 & 2.1 \\
\cline { 2 - 5 } & Total & 36 & 13 & 2.5 \\
\hline \multirow{3}{*}{ More than 6 hours } & Primipara & 2 & 10.1 & 0 \\
\cline { 2 - 5 } & Total & 2 & 10.1 & 0 \\
\hline Total & Primipara & 61 & 15 & 2.8 \\
\cline { 2 - 5 } & Multipara & 59 & 15.3 & 2.4 \\
\cline { 2 - 5 } & Total & 120 & 15.1 & 2.6 \\
\hline
\end{tabular}


The mean score of the duration of the active phase of the first stage of labor in this study was 253.7 minutes (4 hours and 14 minutes) with a standard deviation of 59.3 minutes; at least 2 and up to 6 hours and 20 minutes. Since the active phase of the first stage of labor is affected by delivery rate, the variable was qualitatively ranked for better understanding. Accordingly, the highest frequency in terms of the duration of the active phase of the first stage of labor in nulliparous women was between 5 and 6 hours and it was between 4 and 5 hours in multiparous ones. Considering the correlation between behavioral indicators during labor pain and duration of the active phase of the first stage of labor using Spearman's rank-order correlation test $(\mathrm{p}<0.001$ and $\mathrm{r}=-0.453)$, a comparative study was conducted between the behavioral indicators during labor pain and the active phase of the first stage of labor in nulliparous and multiparous women and, as can be seen, the mean score of behavioral indicators became more desirable as the duration of the first phase of the first stage of labor decreased (Table 3). The results of this study showed that the mean score of the duration of the second stage of labor was 33.7 minutes; with 80 and 5 as maximum and minimum values. With regard to the correlation observed between behavioral indicators during the second stage of labor using Spearman's rank-order correlation ( $\mathrm{p}=0.014$ and $\mathrm{r}=-0.273$ ), the linear regression analysis was employed to predict changes in the scores of the duration of the second stage of labor for variations in mean scores of behavioral indicators during labor pain (Figure 2).

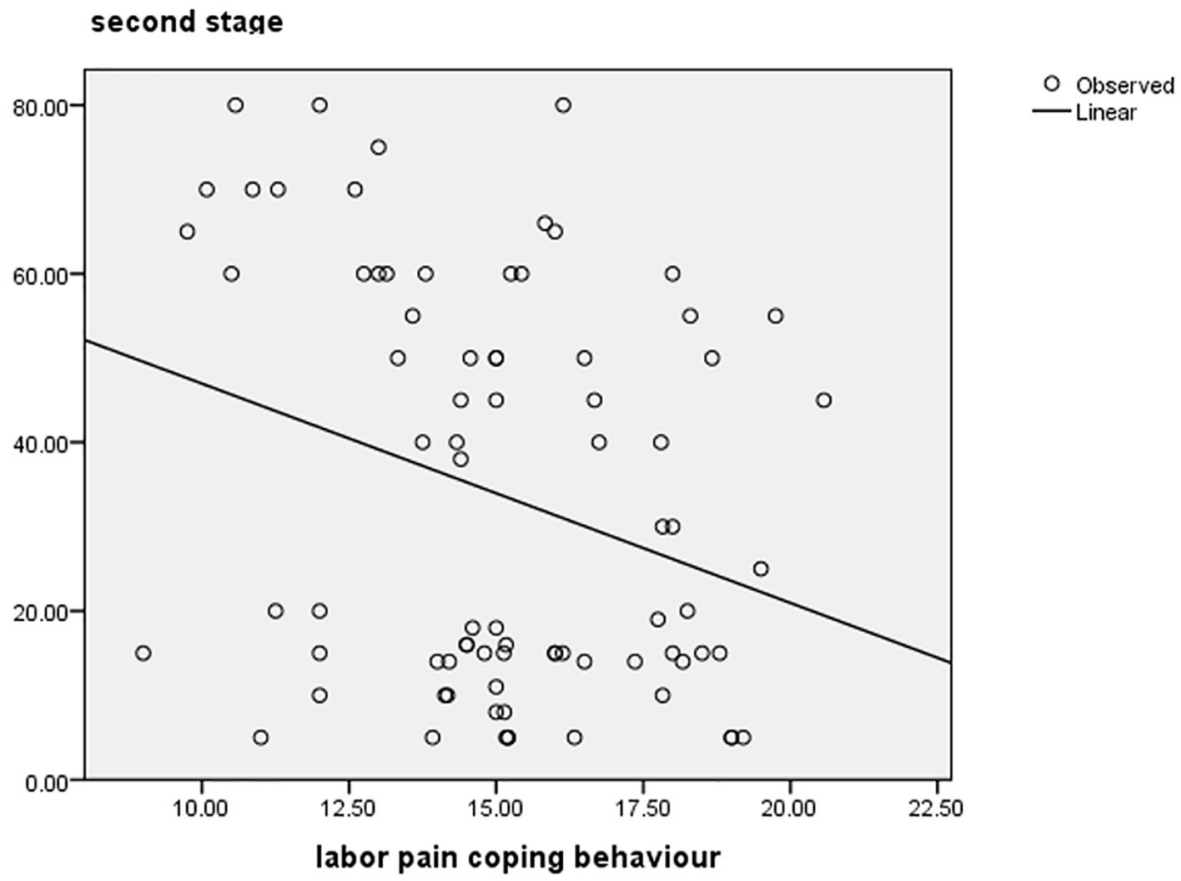

Figure 2. Relationship between mean scores of behavioral indicators during labor pain and duration of the second stage of labor. Linear correlation based on regression tests was as follows: Mean score of labor pain severity = 108.420 - (1.790 × Pain Behavioral Indicators Score $)$

\section{Discussion}

The purpose of the present study was to determine the relationship between behavioral indicators during labor pain, severity of pain, and delivery duration. In the present study, the mean score for the behavioral indicators during labor pain was 15.1 and it was consistent with the results obtained in the study by Tampawiboon (6) in Thailand. In his study, the mean score of behavioral indicators in both control group and the experimental group (the group participating in childbirth preparation classes aimed at teaching correct coping behaviors to deal with labor pain) were compared, in which the mean score of behavioral indicators during labor pain in the control group was equal to 15.2 \pm 2.5 . Moreover, the preliminary comparison showed that the mean scores of behavioral indicators during labor pain in the present study were in line with those obtained in the Tampawiboon's study (6); however, given the high rate of cesarean section in Iran and the inclusion criteria in this study including willingness to have normal vaginal delivery, achieving a reasonable cesarean section rate called for access to more accurate domestic indices. Despite similarities, there is strong evidence that pain experience and perception and response to it are under the influence of socio-cultural context and it is also psychologically and physiologically race-specific (7). Furthermore, there is little evidence regarding the examination of behavioral indicators during labor pain within the literature; thus, no other 
studies in which the behavioral indicators during labor pain had been investigated were found by the researcher for conducting a statistical comparison. Although the mean scores of the behavioral indicators during labor pain in nulliparous and multiparous women were different in the present study, no significant difference was observed between them. The study by Khorsandi (13) also found no significant difference between the score for self-efficacy expectancy and labor pain coping strategies among multiparous and nulliparous women. However, the findings of the study by Jafari (5) revealed a significant relationship between labor pain control and mothers' parity and prior experience with labor. It seems that the successful experience of delivery in multiparous women as participants in this study had not led to incidence of better behaviors during pain. This issue could be due to lack of gaining positive experiences in the course of delivery among these individuals. Since these women had not received any formal education concerning childbirth preparation during their pregnancy and also had passed their previous delivery with fear and pains, this issue could have effects on individuals' judgments about their desirable behaviors to cope with labor pain.

The results of the present study showed that the mean score of pain severity was 81.4 with the highest and the lowest values of 100 and 58.7, respectively; which were in agreement with the findings of other research conducted by Jafari (5), Pirdel (19), and Houshmandi (20). According to the results of this study, the behavioral indicators during labor pain were significantly and inversely correlated with severity of pain. This means that with an increase in the mean score of behavioral indicators during labor pain, a reduction could be observed in severity of pain. In the study by Jafari (5), the control rate of individual behaviors in both experimental and control groups was correlated with pain severity. Moreover, Golmakani found that using desirable behavioral strategies was associated with a lower level of labor pain (21). According to Foroud (22), the use of respiratory patterns to control the severity of labor pain in experimental and control groups showed that emotional behaviors such as loud crying, anxiety and distress, changes in facial expressions, screams, sensitivity, moaning, scratching things, and demonstration of fear in the control group was significantly higher than those among mothers in the experimental group (40\%) compared with the control group (8\%) that had more positive feelings towards labor pain and reported lower levels of severity of pain. In the study by Aliakbari (23) in which the duration of labor stages was studied in experimental and control groups, those endowed with better behaviors to cope with labor pains had experienced lower duration in the first stage of labor, the second stage of the labor, and the whole labor procedure. Aliakbari also argued that; according to the Gate Control Theory of Pain, methods such as relaxation techniques could reduce pain severity perception through moderating pain capacity transmission or blocking the transfer of pain signals. There was also a relationship between adopting some coping strategies and severity of pain in the study by Asghari Moghaddam and Golak (8). Catastrophizing in the face of pain could be similarly accompanied by more severe pain and those with higher selfefficacy in terms of coping with pain had experienced mild levels of pain and also the strategies of pain avoidance and self-talk were associated with less pain. Although the results of this study were somewhat similar to those in the present study, the statistical population and the type of pain were not consistent. The results of these studies indicated that labor pain as a common stressor among women in labor required its own specific behavioral coping. Given the personal perceptions of the situation by women in labor, behavioral indicators during labor pain could be effective or ineffective (15). Adopting harmonious and effective behaviors in the face of pain could also reduce the severity of pain perception, and lead to lower levels of pain. Moreover, undesirable coping behaviors towards stressful and painful situations like screaming and crying, could result in increased oxygen consumption along with physical and psychological fatigue, and undesirable coping complications within a vicious cycle could bring about severe pains (6). Explaining such a possibility, it was stated that a variety of factors including individuals' cognitive structure of pain formed over time (personal experience) could act as an intermediary between type of behavior and severity of pain, and consequently decrease or increase pain (8). According to the results of this study, behavioral indicators during labor pain were significantly and inversely correlated with the duration of the active phase of the first stage of labor as well as the duration of the second stage of labor; this means, the increase in the mean score of behavioral indicators during labor pain could reduce the duration of the first stage and the second stage of labor. The results of this study were consistent with the findings by Tampawiboon (6). In his study; the first, the second, and the third stages of labor were significantly lower in the experimental group. In this respect, Golmakani (21) suggested that using desirable behavioral coping strategies was accompanied by a decline in the duration of labor. Moreover, a relationship was observed between coping strategies and duration of pain in the study by Rezaei et al. (24). This study was also different from the present study considering its study population and severity of pain. However, the most frequently used strategy to control pain by patients was prayers and positivity; the most effective strategy was pain avoidance along with self-talk; and the most ineffective strategy was catastrophizing in terms of pain control. There are also techniques that lead to better behavioral adaptations to pain. Such techniques can be either on the basis of mental functioning such as concentration, creative visualization, and distraction or physical 
methods such as acupressure, reflexology, massage, transcutaneous electrical nerve stimulation (TENS), aromatherapy, music, hypnosis, and other methods, which can all lead to changes in adopting behaviors against labor pain, and the external manifestation of such methods is taking on more efficient and effective behaviors to cope with pain. Among the limitations of this study were the environmental factors affecting mentality which were controlled as much as possible. Moreover, individual differences and mental-psychological conditions of the participants could have effects on responses to questionnaires which were out of the researcher's control. It should be noted that the researcher set the accuracy of the statements made by participants as the criterion.

\section{Conclusions}

Labor pain should be assessed as the fifth vital sign because behaviors demonstrated during delivery have their own specific features and they also have various effects on labor procedure, but the management of labor is rarely based on patients' behaviors and only physiological signs, clinical trials, as well as physiological stages of labor have been taken into account in this respect. Thus, mental and cognitive concepts of labor are lacking in the obstetric care for pregnant women. Behaviors demonstrated by women in labor can also influence labor pain, and such a pain can be correlated with the duration of the stages of labor and severity of labor pain. Accordingly, it is recommended to consider this issue together with physiological symptoms and clinical trials in order to manage women in labor better.

\section{Acknowledgments:}

This study was the result of the thesis approved by the Vice-Chancellor's Office at Mashhad University of Medical Sciences. We, hereby, express our gratitude and appreciate the Office and the Mashhad School of Nursing and Midwifery as well as the personnel of Omolbanin (AS) Hospital for their sincere cooperation.

\section{Conflict of Interest:}

There is no conflict of interest to be declared.

Authors' contributions:

All authors contributed to this project and article equally. All authors read and approved the final manuscript.

\section{References:}

1) Ghanjavi A, Paghande T, Ebrahimi Nejad Gh. The Quality and Intensity of Labor Pain Based on Mc Gill Pain Questionnaire in Parturient Women Admitted in the Maternity Ward of Afzalipour Hospital in Kerman. J Kerman Univ Med Sci. 2010; 18(2): 164.

2) Golmakani N, Hashemi Asl M, Sadjadi A, Ebrahimzade S. Relationship between Happiness during Pregnancy, and labor Pain Coping Behaviors. Evidence based care. 2012; 2(2): 87-95.

3) Roohani Mashhadi S. Effects of SP6 Acupressure on Labor Pain During First Stage of Labor at 17 Shahrivar Hospital in Mashhad in 2005-2006. A Thesis presented for the Master of Science Degree in Midwifery: School of Nursing \& Midwifery of Mashhad. 2006.

4) Gholyan Sh, Nikkhah E, Mehran A, Vasegh F. Effect of relaxation based on meditationtechnique on pain and length of active phase in nulliparous women. Journal of Nursing and Midwifery University of Tehran (hayat). 2006; 12(3): 51-61.

5) Jafari E, Mohebi P, Sedaghatpisheh T, Mazloomzadeh S. Comparison of Personal Control and Its Related Factors, Pain and Labor Outcomes in Physiological and Routine Childbirth Groups. Rafsanjan Univ Med Sci. 2015; 14(12): 1033-48.

6) Tampawiboon K. Effect of Childbirth Preparation on Fear, labor Pain Coping Behaviors and Childbirth Satisfaction in Primiparas. Master's Thesis. Mahdioluniversity in Tailand. 2005.

7) Heidarpour S, Zare E, Mehrabi E, Heidarpour F, Kolivand M. Comparison of primipara's women perception of pain, fear and anxiety of vaginal delivery among Persian, Kurdish and Turkish women. Journal of clinical research in paramedical sciences (JCRPS). 2015; 4(3): 223-30.

8) Asghari Moghaddam M, Gholak N. Role of Coping strategies with pain in coping with chronic pain. Daneshvarraftar. 2005; 12(10): 1-23.

9) Mirzai A. The Effect of Communication Skills Training on Nursing Students Coping Strategies in Stressful Situations of Clinical Courses. A Thesis Presented for the Master of Science Degree in Nursing: School of Nursing \&Midwifery of Mashhad. 2009.

10) Brunner Lillian Shultis. Pain Treatment, Health Check, Critical Thinking, Ethical Decision And the Nursing Process (Brunnere\&Suddarth). Sami Puran. Tehran: Boshra; 2014; 18-9. 
11) Kushan M, Vaghei S. Psychiatric Nursing (Mental Health1). Tehran. AndisheRafia Publisher. 2007; 91 -3.

12) Kamali S, Ahmari H, Mohammadalizade S, Jafari E. Effect of supportive behaviors on the process of labor in nulliparous women. Qom University of Medical Sciences. 2010; 4(2): 1-14.

13) Khorsandy M, Ghofranipur F, Heidarnia A, Faghihzade S, Akbarzade A, Vafai M. Self-efficacy for Labor and Childbirth in Pregnant Women. Medical Council of Islamic Republic of Iran. 2008; 26(1): 89-95.

14) Rastegari L, Mohebbi P, Mazlomzadeh S. The Effect of Childbirth Preparation Training Classes on Perceived Self-efficacy in Delivery of Pregnant Women. Zanjan Univ Med Sci. 2013; 21(86): 105-15.

15) Abushaikha LA. Methods of Coping with Labor Pain Used by Jordanian Women. J Transcult Nurs. 2007; 18(1): 35-40. doi: 10.1177/1043659606294194. PMID: 17202527.

16) Dadipur S, Madani A, Alavi A, Rouzbeh N, Moradabadi A. A Survey of Growing Cesarean Rate in Iran and the World: Review Article. The Iranian Journal of Obstetrics, Gynecology and Infertility. 2016; 19(27): 8-17.

17) Sodsang W. The Effects of Nursing Support During Labour on Labour Pain Coping Behaviours and the Perception of Childbirth Experience of First-time Mothers. Master's Thesis. Mahdioluniversity in Tailand; 2005.

18) Sindamrong S. Marital Relationship, Self-efficacy in Labor Pain Coping. And Pain Coping Behavior During Labor of the First Time Mothers. Master's Thesis. Mahdioluniversity in Tailand; 2004

19) Pirdel M, Kamifard M, Bani S, Ghoujazadeh M. Comparison of labor pain and effective factors on labor pain perception in primiparous and multiparous women Referring to Alzahra Tabriz Medical Center. Tabriz Nursing and Midwifery Journal. 2006; 5: 37-46.

20) Houshmandi S, Dolatian M, Kamifard M, Ghoujazadeh M. Comparison of the labor pain intensity and the factors affecting its perception amongnulliparous and multiparous womeninpublicand privatehospital. Medical Journal of Tabriz Medical University. 2012; 34(3): 117-21.

21) Golmakani N, Hashemiasl M, Sadjadi A. survey of Related factor with labor pain coping behaviors. The Iranian Journal of Obstetrics, Gynecology and Infertility. 2012; 15(24): 17-25.

22) Forud A, Mahdipur S. Effect of breathing patterns and massage on Attitude and Intensity of pain in nulliparous women. Shahrkord University of Medical Sciences. 2005; 7(4): 70-7.

23) Aliakbari S, Jamalian R, Khohan Sh, Valai N. Impact of childbirth preparation on labor pain and Length of delivery time. Journal of Feiz. 2001; 15: 45.

24) Rezai S, Afsharnejad T, Kafi M, Soltani R, Fallahkohan S. Relationship between depression and coping strategy with chronic back pain: a controlled study of pain intensity and duration. Bimonthly scienceResearch of Shahed University (Daneshvarraftar). 2009; 16(81): 63-74. 